\title{
Pseudo-Hermiticity for a class of nondiagonalizable Hamiltonians
}

Ali Mostafazadeh

Citation: Journal of Mathematical Physics 43, 6343 (2002); doi: 10.1063/1.1514834

View online: http://dx.doi.org/10.1063/1.1514834

View Table of Contents: http://aip.scitation.org/toc/jmp/43/12

Published by the American Institute of Physics

\section{Articles you may be interested in}

Pseudo-Hermiticity versus PT symmetry: The necessary condition for the reality of the spectrum of a nonHermitian Hamiltonian

Journal of Mathematical Physics 43, 205 (2001); 10.1063/1.1418246

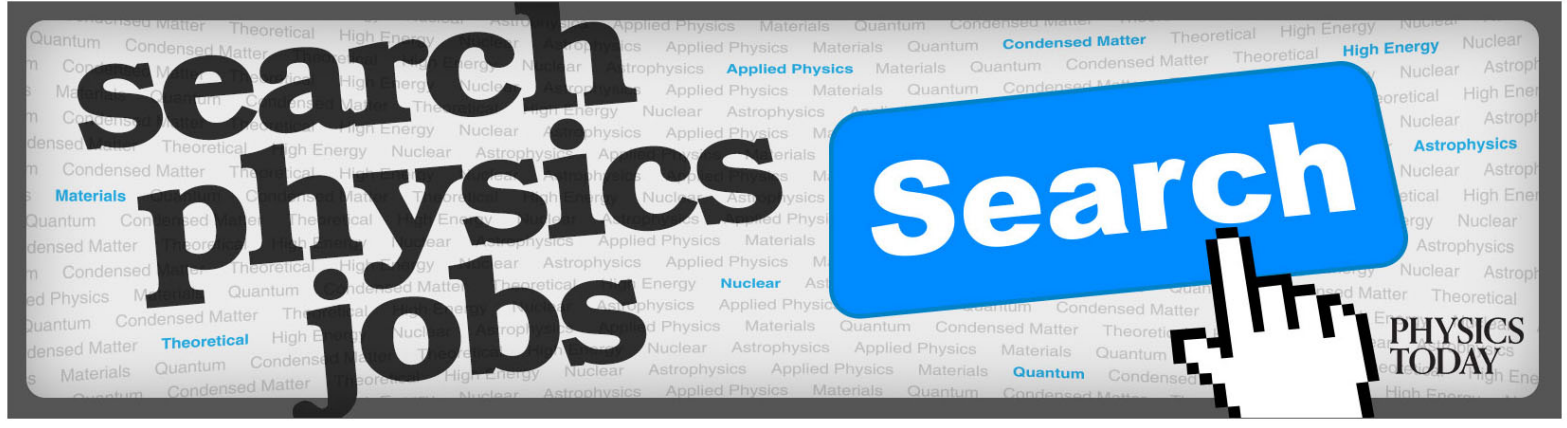




\title{
Pseudo-Hermiticity for a class of nondiagonalizable Hamiltonians
}

\author{
Ali Mostafazadeh ${ }^{\text {a) }}$ \\ Department of Mathematics, Koç University, \\ Rumelifeneri Yolu, 80910 Sariyer, Istanbul, Turkey
}

(Received 8 July 2002; accepted 12 August 2002)

\begin{abstract}
We give two characterization theorems for pseudo-Hermitian (possibly nondiagonalizable) Hamiltonians with a discrete spectrum that admit a block-diagonalization with finite-dimensional diagonal blocks. In particular, we prove that for such an operator $H$ the following statements are equivalent: (1) $H$ is pseudo-Hermitian; (2) the spectrum of $H$ consists of real and/or complex-conjugate pairs of eigenvalues and the geometric multiplicity and the dimension of the diagonal blocks for the complex-conjugate eigenvalues are identical; (3) $H$ is Hermitian with respect to a positive-semidefinite inner product. We further discuss the relevance of our findings for the merging of a complex-conjugate pair of eigenvalues of diagonalizable pseudo-Hermitian Hamiltonians in general, and the PT-symmetric Hamiltonians and the effective Hamiltonian for a certain closed FRW minisuperspace quantum cosmological model in particular. (C) 2002 American Institute of Physics.
\end{abstract}

[DOI: $10.1063 / 1.1514834]$

\section{INTRODUCTION}

In Refs. 1-5 we developed the notion of a pseudo-Hermitian operator and investigated its various consequences in particular in connection with PT-symmetric quantum systems ${ }^{6}$ and twocomponent formulation of the FRW minisuperspace quantum cosmology. ${ }^{7}$ Since the announcement of the results of Ref. 1 several authors have explored the implications of pseudo-Hermiticity. ${ }^{8}$ The main results reported in Refs. $1-5$ were, however, based on the assumption that the Hamiltonian of the system is diagonalizable and has a discrete spectrum. As demonstrated in Ref. 5, the latter condition can be easily relaxed. Moreover, in Ref. 9 we showed, without making any assumption about the diagonalizability of the Hamiltonian or discreteness of its spectrum, that the results of Refs. 1 and 3 generalized to the class of all PT-symmetric standard Hamiltonians having $\mathbb{R}$ as their configuration space. This suggests that these results may be valid under more general conditions. Our purpose in the present article is to generalize the results of Ref. 1 to the class of possibly nondiagonalizable Hamiltonians that admit a block-diagonalization with finite-dimensional diagonal blocks. This, in particular, includes all the matrix Hamiltonians. It is also relevant to the accidental loss of diagonalizability due to the pseudo-Hermiticitypreserving variations of diagonalizable pseudo-Hermitian Hamiltonians that lead to the merging of complex-conjugate pairs of eigenvalues.

The organization of the article is as follows. In Sec. II, we discuss the basic properties of the class of the Hamiltonians admitting a block-diagonalization with finite-dimensional diagonal blocks. In Sec. III, we present two characterization theorems for pseudo-Hermitian Hamiltonians belonging to this class. In Sec. IV, we study general $2 \times 2$ matrix Hamiltonians. In Sec. V, we discuss an application of our results in quantum cosmology. Finally, in Sec. VI, we present our concluding remarks.

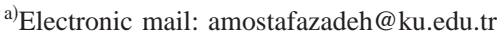




\section{BLOCK-DIAGONALIZABLE HAMILTONIANS WITH FINITE-DIMENSIONAL DIAGONAL BLOCKS}

Consider a linear operator $H: \mathcal{H} \rightarrow \mathcal{H}$ acting in a (separable) Hilbert space $\mathcal{H}$ and having a discrete spectrum. Suppose that for every eigenvalue $E_{n}$, there are positive integers $g_{n}, p_{n} \in Z^{+}$ such that for all $l \in \mathbb{Z}^{+}$,

$$
d_{n, l}:=\operatorname{dim}\left[\operatorname{ker}\left(H-E_{n} 1\right)^{l}\right]=g_{n}, \text { if and only if } l \geqslant p_{n} .
$$

This in particular means that

$$
d_{n, 1} \leqslant d_{n, 2} \leqslant \cdots \leqslant d_{n, p_{n}-1} \leqslant d_{n, p_{n}}=g_{n} .
$$

The integer $d_{n, 1}$ is just the degree of degeneracy or the geometric multiplicity of $E_{n}$. In what follows, we shall use the abbreviated notation $d_{n}$ for $d_{n, 1}$ and denote the degeneracy labels $1,2, \ldots, d_{n}$ by the letters from the beginning of the Latin alphabet.

The integer $g_{n}$ is called the algebraic multiplicity of $E_{n}$. The condition (1) means that all the eigenvalues of $H$ have finite algebraic multiplicity. Throughout this paper we shall assume that this condition is satisfied and that there is a basis of the Hilbert space in which $H$ is block-diagonal with diagonal blocks being finite-dimensional. In this case, we can always find a basis in which the diagonal blocks have the canonical Jordan form, ${ }^{10}$ i.e., there is an invertible operator $A: \mathcal{H} \rightarrow \mathcal{H}$ and an orthonormal basis $\{|n, a, i\rangle\}$ with $n$ being the spectral label, $a \in\left\{1,2, \ldots, d_{n}\right\}, i$ $\in\left\{1,2, \ldots, p_{n, a}\right\}$, and $p_{n, a} \in \mathbb{Z}^{+}$, such that

$$
A^{-1} H A=H_{b}:=\sum_{n} \sum_{a=1}^{d_{n}}\left(E_{n} \sum_{i=1}^{p_{n, a}}|n, a, i\rangle\left\langle n, a, i\left|+\sum_{i=1}^{p_{n, a}-1}\right| n, a, i\right\rangle\langle n, a, i+1|\right) .
$$

Alternatively, letting

$$
\left|\psi_{n}, a, i\right\rangle:=A|n, a, i\rangle,\left|\phi_{n}, a, i\right\rangle:=A^{-1 \dagger}|n, a, i\rangle,
$$

we have

$$
\begin{gathered}
\left\langle\psi_{n}, a, i \mid \phi_{m}, b, j\right\rangle=\delta_{m n} \delta_{a b} \delta_{i j}, \quad \sum_{i} \sum_{a=1}^{d_{n}} \sum_{i=1}^{p_{n, a}}\left|\psi_{n}, a, i\right\rangle\left\langle\phi_{n}, a, i\right|=1, \\
H=A H_{b} A^{-1}=\sum_{n} \sum_{a=1}^{d_{n}}\left(E_{n} \sum_{i=1}^{p_{n, a}}\left|\psi_{n}, a, i\right\rangle\left\langle\phi_{n}, a, i\left|+\sum_{i=1}^{p_{n, a}-1}\right| \psi_{n}, a, i\right\rangle\left\langle\phi_{n}, a, i+1\right|\right) .
\end{gathered}
$$

Note that according to Eqs. (5) and (6), $\left\{\left|\psi_{n}, a, i\right\rangle,\left|\phi_{n}, a, i\right\rangle\right\}$ is a complete biorthonormal system for the Hilbert space and

$$
\begin{gathered}
H\left|\psi_{n}, a, 1\right\rangle=E_{n}\left|\psi_{n}, a, 1\right\rangle, \\
H^{\dagger}\left|\phi_{n}, a, p_{n, a}\right\rangle=E_{n}^{*}\left|\phi_{n}, a, p_{n, a}\right\rangle .
\end{gathered}
$$

Hence $\left|\psi_{n}, a, 1\right\rangle$ are the eigenvectors of $H$ and $\left|\phi_{n}, a, p_{n, a}\right\rangle$ are the eigenvectors of $H^{\dagger}$.

The numbers $p_{n, a}$ represent the dimension of the Jordan block associated with the spectral label $n$ and the degeneracy label $a$. We shall refer to them as the Jordan dimensions. For a given eigenvalue $E_{n}$, the number of the corresponding Jordan blocks (which is equal to the geometric multiplicity of $E_{n}$ ) and the Jordan dimensions are uniquely determined by the integers $d_{n, l}$ of (1) up to the permutations of the degeneracy labels. ${ }^{10}$ Note also that the algebraic multiplicity is the sum of the Jordan dimensions, $g_{n}:=\sum_{a=1}^{d_{n}} p_{n, a}$. 


\section{CONSEQUENCES OF PSEUDO-HERMITICITY}

Theorem 1: Let $H: \mathcal{H} \rightarrow \mathcal{H}$ be a linear operator acting in a (separable) Hilbert space $\mathcal{H}$. Suppose that the spectrum of $H$ is discrete, its eigenvalues have finite algebraic multiplicity, and that (6) holds. Then, $H$ is pseudo-Hermitian if and only if the eigenvalues of $H$ are either real or come in complex-conjugate pairs and the geometric multiplicity and the Jordan dimensions of the complex-conjugate eigenvalues coincide.

Proof: Suppose that $H$ is pseudo-Hermitian. Then, by definition, ${ }^{1}$ there is a Hermitian automorphism (linear bijection mapping $\mathcal{H}$ onto $\mathcal{H}) \eta: \mathcal{H} \rightarrow \mathcal{H}$ such that $H^{\dagger}=\eta H \eta^{-1}$. Now let $E_{n}$ be an arbitrary element of the spectrum of $H$. Then, by virtue of Eqs. (7) and (8), for each $a$ $\in\left\{1,2, \ldots, d_{n}\right\},\left|\psi_{n}, a, 1\right\rangle$ is an eigenvector of $H$ with eigenvalue $E_{n}$ and $\left|\phi_{n}, a, p_{n, a}\right\rangle$ is an eigenvector of $H^{\dagger}$ with eigenvalue $E_{n}^{*}$. This in turn implies $H \eta^{-1}\left|\phi_{n}, a, p_{n, a}\right\rangle=\eta^{-1} H^{\dagger}\left|\phi_{n}, a, p_{n, a}\right\rangle$ $=E_{n}^{*} \eta^{-1}\left|\phi_{n}, a, p_{n, a}\right\rangle$. As $\eta^{-1}$ is an invertible operator, $\eta^{-1}\left|\phi_{n}, a, p_{n, a}\right\rangle \neq 0$. Hence $E_{n}^{*}$ also belongs to the spectrum of $H$. Next, note that because the eigenvalues of $H$ and consequently $H^{\dagger}$ have finite algebraic multiplicity, for every $l \in \mathbb{Z}^{+}, \operatorname{kernel}\left(H-E_{n}\right)^{l}, \operatorname{kernel}\left(H-E_{n}^{*}\right)^{l}$, and $\operatorname{kernel}\left(H^{\dagger}-E_{n}^{*}\right)^{l}$ are finite-dimensional subspaces of $\mathcal{H}$. Clearly, as a result of (6), $H$ and $H^{\dagger}$ have essentially the same Jordan block-diagonalization. In particular, the geometric multiplicity and the Jordan dimensions of $E_{n}^{*}$ as an eigenvalue of $H^{\dagger}$ is the same as the geometric multiplicity and the Jordan dimensions of $E_{n}$ as an eigenvalue of $H$. This implies that $\operatorname{kernel}\left(H^{\dagger}-E_{n}^{*}\right)^{l}$ and $\operatorname{kernel}\left(H-E_{n}\right)^{l}$ have the same dimension. Thus they are isomorphic. Furthermore, using the fact that $\eta$ is an automorphism, $\operatorname{kernel}\left(H^{\dagger}-E_{n}^{*}\right)^{l}$ is also isomorphic to

$$
\operatorname{kernel}\left[\eta^{-1}\left(H^{\dagger}-E_{n}^{*}\right)^{l} \eta\right]=\operatorname{kernel}\left(\eta^{-1} H^{\dagger} \eta-E_{n}^{*}\right)^{l}=\operatorname{kernel}\left(H-E_{n}^{*}\right)^{l} .
$$

Therefore, for every $l \in Z^{+}, \operatorname{kernel}\left(H-E_{n}\right)^{l}$ and $\operatorname{kernel}\left(H-E_{n}^{*}\right)^{l}$ are isomorphic and consequently have the same dimension. This in turns implies that the number of the Jordan blocks associated with $E$ and their dimensions are identical with those of $E_{n}^{*}$, i.e., $E_{n}$ and $E_{n}^{*}$ have the same geometric multiplicity, and up to permutations of the degeneracy labels they have identical Jordan dimensions as well. Conversely, suppose that the eigenvalues of $H$ are either real or come in complex-conjugate pairs and the geometric multiplicity $d_{n}$ and the Jordan dimensions $p_{n, a}$ of the complex conjugate pairs of eigenvalues are identical. We shall set $n=\nu_{0}, \nu, \nu-$ depending on whether imaginary part of $E_{n}$ is zero, positive, or negative. Then $E_{\nu^{-}}=E_{\nu}^{*}, d_{\nu^{-}}=d_{\nu}$, for all $a$ $\in\left\{1,2, \ldots, d_{n}\right\}, p_{\nu-, a}=p_{\nu, a}$, and Eq. (6) takes the form

$$
\begin{aligned}
H= & \sum_{\nu_{0}} \sum_{a=1}^{d_{\nu_{0}}}\left(E_{\nu_{0}} \sum_{i=1}^{p_{\nu_{0}, a}}\left|\psi_{\nu_{0}}, a, i\right\rangle\left\langle\phi_{\nu_{0}}, a, i\left|+\sum_{i=1}^{p_{\nu_{0}, a}-1}\right| \psi_{\nu_{0}}, a, i\right\rangle\left\langle\phi_{\nu_{0}}, a, i+1\right|\right) \\
& +\sum_{\nu} \sum_{a=1}^{d_{\nu}}\left[\sum_{i=1}^{p_{\nu, a}}\left(E_{\nu}\left|\psi_{\nu}, a, i\right\rangle\left\langle\phi_{\nu}, a, i\left|+E_{\nu}^{*}\right| \psi_{\nu-}, a, i\right\rangle\left\langle\phi_{\nu^{-}}, a, i\right|\right)\right. \\
& \left.+\sum_{i=1}^{p_{\nu, a}-1}\left(\left|\psi_{\nu}, a, i\right\rangle\left\langle\phi_{\nu}, a, i+1|+| \psi_{\nu-}, a, i\right\rangle\left\langle\phi_{\nu^{-}}, a, i+1\right|\right)\right] .
\end{aligned}
$$

Next, let

$$
\begin{aligned}
\eta(x, \xi):= & \sum_{\nu_{0}} \sum_{a=1}^{d_{\nu_{0}}} \sum_{i=1}^{p_{\nu_{0}, a}} \sum_{j=p_{\nu_{0}, a}+1-i}^{p_{\nu_{0}, a}} x_{\nu_{0}, a, i+j}\left|\phi_{\nu_{0}}, a, i\right\rangle\left\langle\phi_{\nu_{0}}, a, j\right| \\
& +\sum_{\nu} \sum_{a=1}^{d_{\nu}} \sum_{i=1}^{p_{\nu, a}} \sum_{j=p_{\nu, a}+1-i}^{p_{\nu, a}}\left(\xi_{\nu, a, i+j}\left|\phi_{\nu}, a, i\right\rangle\left\langle\phi_{\nu-}, a, j\left|+\xi_{\nu, a, i+j}^{*}\right| \phi_{\nu^{-}}, a, j\right\rangle\left\langle\phi_{\nu}, a, i\right|\right),
\end{aligned}
$$

where $x_{\nu_{0}, a, k} \in \mathbb{R}, \xi_{\nu, a, k} \in \mathbb{C}$, 


$$
x_{\nu_{0}, a, p_{\nu_{0}, a}+1} \neq 0 \neq \xi_{\nu, a, p_{\nu, a}+1},
$$

and $x$ and $\xi$, respectively, stand for the sequences $\left\{x_{\nu_{0}, a, k}\right\}$ and $\left\{\xi_{\nu, a, k}\right\}$. It is not difficult to check that, for all $n=\nu_{0}, \nu, \nu-, m=\mu_{0}, \mu, \mu-$, and the corresponding degeneracy labels $a, b$ and Jordan block labels $i, j$,

$$
\begin{aligned}
\left\langle\psi_{\nu_{0}}, a, i|\eta(x, \xi)| \psi_{\mu_{0}}, b, j\right\rangle & =\left\{\begin{array}{l}
\delta_{\nu_{0}, \mu_{0}} \delta_{a b} x_{\nu_{0}, a, i+j}, \quad \text { for } i+j>p_{\nu_{0}, a} \\
0, \quad \text { otherwise }
\end{array}\right. \\
\left\langle\psi_{\nu}, a, i|\eta(x, \xi)| \psi_{\mu-}, b, j\right\rangle & =\left\langle\psi_{\mu-}, a, i|\eta(x, \xi)| \psi_{\nu}, b, j\right\rangle^{*} \\
& = \begin{cases}\delta_{\nu, \mu} \delta_{a b} \xi_{\nu, a, i+j}, & \text { for } i+j>p_{\nu, a} \\
0, & \text { otherwise; }\end{cases}
\end{aligned}
$$

and that the other matrix elements of $\eta:=\eta(x, \xi)$, in the basis $\left\{\left|\psi_{n}, a, j\right\rangle\right\}$, vanish. In view of Eqs. (12), (13), and (11), $\eta$ is a Hermitian automorphism. Furthermore, using Eqs. (5) and (9)-(11), one can check that it satisfies $\eta H=H^{\dagger} \eta$. Hence, $H^{\sharp}:=\eta^{-1} H^{\dagger} \eta=H$, and $H$ is $\eta$-pseudo-Hermitian.

An immediate consequence of this theorem is the following

Corollary 1: Let $H$ be as in Theorem 1. Then the pseudo-Hermiticity of $H$ is a necessary condition for the reality of its spectrum.

Note that (10) is not the most general expression for an $\eta$ with respect to which $H$ is $\eta$-pseudo-Hermitian. One can obtain more general expressions by performing appropriate basis transformations. (These are the transformations that mix the basis vectors with different degeneracy labels $a$ but identical spectral label $n$ and the Jordan dimension $p_{n, a}$ ). Similarly to the diagonalizable case, ${ }^{4}$ one can also perform a change of basis to set $x_{\nu_{0}, a, k}= \pm 1$ and $\xi_{\nu, a, k}=1$. This is, however not the simplest choice for $\eta$. It is not difficult to check that the following simpler choice works as well:

$$
x_{\nu_{0}, a, k}=\left\{\begin{array}{l} 
\pm 1, \quad \text { for } k=p_{\nu_{0}, a}+1, \\
0, \quad \text { otherwise },
\end{array} \xi_{\nu, a, k}= \begin{cases}1, & \text { for } k=p_{\nu, a}+1 \\
0, & \text { otherwise }\end{cases}\right.
$$

In this way one obtains the following set of simple canonical automorphisms with respect to which $H$ is $\eta$-pseudo-Hermitian:

$$
\begin{aligned}
\eta(\sigma):= & \sum_{\nu_{0}} \sum_{a=1}^{d_{\nu_{0}}} \sigma_{\nu_{0}, a}\left(\sum_{i=1}^{p_{\nu_{0}}, a}\left|\phi_{\nu_{0}}, a, i\right\rangle\left\langle\phi_{\nu_{0}}, a, p_{\nu_{0}, a}+1-i\right|\right) \\
& +\sum_{\nu} \sum_{a=1}^{d_{\nu}} \sum_{i=1}^{p_{\nu, a}}\left(\left|\phi_{\nu}, a, i\right\rangle\left\langle\phi_{\nu-}, a, p_{\nu, a}+1-i|+| \phi_{\nu-}, a, p_{\nu, a}+1-i\right\rangle\left\langle\phi_{\nu}, a, i\right|\right)
\end{aligned}
$$

with $\sigma:=\left\{\sigma_{\nu_{0}, a}\right\}$ being a sequence of signs. A straightforward calculation shows that

$$
\begin{aligned}
\eta(\sigma)^{-1}:= & \sum_{\nu_{0}} \sum_{a=1}^{d_{\nu_{0}}} \sigma_{\nu_{0}, a}\left(\sum_{i=1}^{p_{\nu_{0}, a}}\left|\psi_{\nu_{0}}, a, i\right\rangle\left\langle\psi_{\nu_{0}}, a, p_{\nu_{0}, a}+1-i\right|\right) \\
& +\sum_{\nu} \sum_{a=1}^{d_{\nu}} \sum_{i=1}^{p_{\nu, a}}\left(\left|\psi_{\nu}, a, i\right\rangle\left\langle\psi_{\nu^{-}}, a, p_{\nu, a}+1-i|+| \psi_{\nu-}, a, p_{\nu, a}+1-i\right\rangle\left\langle\psi_{\nu}, a, i\right|\right) .
\end{aligned}
$$


If $H$ is diagonalizable, $p_{n, a}=1$ and (15) yields the expression for the canonical automorphisms given in Ref. 4. Again choosing all the signs $\sigma_{\nu_{0}, a}$ to be positive yields a positivesemidefinite (non-negative) $\eta$ and a positive-semidefinite inner product,

$$
\langle\langle\psi, \phi\rangle\rangle_{\eta}:=\langle\psi|\eta| \phi\rangle .
$$

However, even if the complex eigenvalues are absent this choice does not lead to a positivedefinite inner product on the Hilbert space unless $H$ is diagonalizable. This is because in general there are defective (real) eigenvalues $E_{\nu_{0}}$; at least one of the Jordan dimensions $p_{\nu_{0}, a}$ is greater than 1; and according to (12) and (13), $\left\langle\left\langle\psi_{\nu_{0}}, a, 1 \mid \psi_{\nu_{0}}, a, 1\right\rangle\right\rangle_{\eta}=\left\langle\psi_{\nu_{0}}, a, 1|\eta| \psi_{n u_{0}}, a, 1\right\rangle=0$. Hence the corresponding eigenvector $\left|\psi_{\nu_{0}}, a, 1\right\rangle$ is null, and the inner product (17) is not positive-definite.

Theorem 2: Let $H$ be as in Theorem 1. Then $H$ is pseudo- Hermitian if and only if it is Hermitian with respect to a positive-semidefinite inner product $\langle\langle\rangle\rangle:, \mathcal{H}^{2} \rightarrow \mathrm{C}$, i.e., for all $\phi, \psi$ $\in \mathcal{H},\langle\langle\phi, H \psi\rangle\rangle=\langle\langle H \phi, \psi\rangle\rangle$.

Proof: Suppose $H$ is pseudo-Hermitian, then according to Theorem 1 it has real and/or complex-conjugate pairs of eigenvalues with identical geometric multiplicity and Jordan dimensions. According to the proof of this theorem, this implies that $H$ is pseudo-Hermitian with respect to the automorphism (15) with $\sigma_{\nu_{0}, a}=1$ for all $\nu_{0}$ and $a \in\left\{1,2, \ldots, d_{\nu_{0}}\right\}$. The latter yields the positive-semidefinite inner product (17) which satisfies, for all $\psi, \phi \in \mathcal{H}$,

$$
\langle\langle\phi, H \psi\rangle\rangle_{\eta}=\langle\phi|\eta H| \psi\rangle=\left\langle\phi\left|H^{\dagger} \eta\right| \psi\right\rangle=\langle H \phi|\eta| \psi\rangle=\langle\langle H \phi, \psi\rangle\rangle_{\eta} .
$$

Hence $H$ is Hermitian with respect to the inner product (17). Conversely, let $H$ be Hermitian with respect to a positive-semidefinite inner product $\langle\langle\rangle$,$\rangle . Let \eta: \mathcal{H} \rightarrow \mathcal{H}$ be defined in terms of its matrix elements according to, for all $\psi, \phi \in \mathcal{H}$,

$$
\langle\psi|\eta| \phi\rangle:=\langle\langle\psi, \phi\rangle\rangle .
$$

Then, because $\left\langle\langle\right.$,$\rangle is a sesquilinear, Hermitian, nondegenerate quadratic form, { }^{11} \eta$ is a linear, Hermitian, automorphism. Furthermore, because $H$ is Hermitian with respect to $\langle\langle$,$\rangle we have, for$ all $\psi, \phi \in \mathcal{H}$,

$$
\langle\phi \mid \eta H \psi\rangle=\langle\langle\phi, H \psi\rangle\rangle=\langle\langle H \phi, \psi\rangle\rangle=\langle H \phi|\eta| \psi\rangle=\left\langle\phi \mid H^{\dagger} \eta \psi\right\rangle .
$$

Therefore, $\eta H=H^{\dagger} \eta$ or $H^{\sharp}:=\eta^{-1} H^{\dagger} \eta=H$, i.e., $H$ is pseudo-Hermitian.

\section{IV. $2 \times 2$ MATRIX HAMILTONIANS}

In Ref. 3, we showed that the pseudo-Hermiticity of a diagonalizable Hamiltonian is equivalent to the presence of antilinear symmetries. The PT-symmetry studied in the literature ${ }^{6}$ is a primary example. In general, such a Hamiltonian depends on certain continuous parameters whose variation does not destroy the symmetry but changes the spectrum. In particular, it is possible that under such variations complex-conjugate pairs of eigenvalues merge and produce real eigenvalues or a real eigenvalue splits into a complex-conjugate pair of eigenvalues. This is a generic behavior observed in the numerical studies of PT-symmetric Hamiltonians ${ }^{6}$ and naturally applies in the case of general pseudo-Hermitian Hamiltonians. Now consider a diagonalizable pseudo-Hermitian Hamiltonian with a discrete spectrum that undergoes a continuous pseudo-Hermiticity-preserving perturbation. In general, such a perturbation may not preserve the diagonalizability of the Hamiltonian. ${ }^{12}$ In particular, at the critical values of the perturbation parameter when two nondegenerate complex-conjugate eigenvalues merge to produce a real eigenvalue, there is no guarantee that the resulting eigenvalue is doubly degenerate. This observation underlies the importance of the results of Sec. III in the study of the behavior of diagonalizable pseudo-Hermitian operators undergoing arbitrary pseudo-Hermiticity-preserving perturbations. 
Consider the case that under such a perturbation a pair of complex-conjugate nondegenerate eigenvalues cross while no other level-crossing occurs. In the vicinity of this level-crossing, one can approximate the behavior of the Hamiltonian by a traceless $2 \times 2$ matrix Hamiltonian. In Ref. 4 , we have studied the properties of general complex, traceless, diagonalizable, pseudo-Hermitian $2 \times 2$ matrix Hamiltonians. A traceless $2 \times 2$ matrix $H$ with two nondegenerate eigenvalues is pseudo-Hermitian if its determinant is a nonzero real number. ${ }^{4}$ As we explain below the converse of this statement is also true. In particular, $\operatorname{det}(H)<0 \operatorname{or} \operatorname{det}(H)>0$ depending on whether the eigenvalues are real or imaginary. This means that the moduli space $\mathcal{M}$ of traceless pseudoHermitian $2 \times 2$ matrices with two nondegenerate eigenvalues is a 5-dimensional subspace of the 8 -dimensional space $M(2, \mathrm{C})$ of all complex $2 \times 2$ matrices. The latter has the manifold structure of $\mathrm{C}^{4}=\mathrm{R}^{8}$. If we, respectively, denote the subsets of complex traceless $2 \times 2$ matrices, complex traceless pseudo-Hermitian $2 \times 2$ matrices, and traceless Hermitian $2 \times 2$ matrices by $M_{0}, \mathcal{M}^{\prime}$, and $\mathcal{M}_{0}$, we have

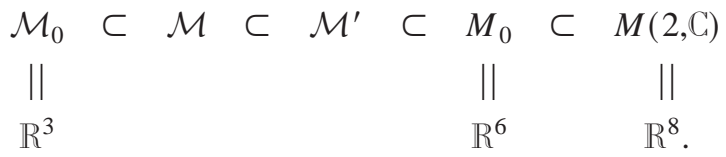

We can identify $\mathcal{M}$ with the inverse image of $R-\{0\} \subset \mathbb{R}^{2}=\mathrm{C}$ under the continuous function $\operatorname{det}: M(2, \mathrm{C}) \rightarrow \mathrm{C}=\mathbb{R}^{2}$. Noting that $\mathrm{R}^{+}$and $\mathbb{R}^{-}$are disjoint, open, connected subsets of $\mathrm{C}$ and det is continuous, we infer that $\mathcal{M}$ consists of two open connected components, namely

$$
\mathcal{M}^{ \pm}:=\left\{H \in \mathcal{M} \mid \operatorname{det} H \in \mathbb{R}^{ \pm}\right\} .
$$

This in turn implies that at a critical point of the parameters of $H$ where a level-crossing happens, $H$ fails to stay in $\mathcal{M}$. This is also easily seen by realizing that because $H$ is traceless, a levelcrossing can occur only if $\operatorname{det} H$ vanishes. Therefore, at the level-crossing either $H=0$ or it is nondiagonalizable.

In fact, it is not difficult to see that an element $X_{ \pm}$of $\mathcal{M}^{ \pm}$has the general form

$$
X_{ \pm}=\sqrt{ \pm 1} E g^{-1} \sigma_{3} g,
$$

where $E$ is a nonzero real number, $g$ is an element of the special linear group $S L(2, \mathrm{C})$, and $\sigma_{3}$ is the diagonal Pauli matrix $\operatorname{diag}(1,-1)$. The form (18) indicates that the moduli spaces $\mathcal{M}^{+}$and $\mathcal{M}^{-}$have the manifold structure of $F \times(\mathbb{R}-\{0\})$ where $F$ is the 4-dimensional homogeneous space:

$$
F:=S L(2, \mathrm{C}) / U_{\mathrm{C}}(1),
$$

and

$$
U_{\mathrm{C}}(1):=\left\{e^{z \sigma_{3}} \mid z \in \mathrm{C}\right\}=\left\{\left(\begin{array}{cc}
w & 0 \\
0 & w^{-1}
\end{array}\right) \mid w \in \mathrm{C}-\{0\}\right\} .
$$

Furthermore, according to (18) the group elements $g$ that are uniquely parametrized by the points of $F$ play the same role for both $X_{+}$and $X_{-}$. It is the factor $\sqrt{ \pm 1} E$ in (18) that differentiates $X_{+}$ and $X_{-}$. This suggests that we can identify $\mathcal{M}^{ \pm}$by $F \times L^{ \pm}$, where

$$
\begin{gathered}
L^{+}:=\{z \in \mathrm{C}-\{0\} \mid \operatorname{Re}(z)=0\}=\text { imaginary axis in the complex plane with } 0 \text { removed, } \\
L^{-}:=\{z \in \mathrm{C}-\{0\} \mid \operatorname{Im}(z)=0\}=\text { real axis in the complex plane with } 0 \text { removed, }
\end{gathered}
$$

and "Re" and "Im" stand for the "real" and the "imaginary" part of the corresponding complex variable, respectively. 
The above picture of $\mathcal{M}$ confirms our earlier remark that at a level-crossing a traceless pseudo-Hermitian $2 \times 2$ matrix,

$$
H=\left(\begin{array}{cc}
a & b \\
c & -a
\end{array}\right)
$$

either vanishes identically:

$$
a=b=c=0,
$$

or becomes nondiagonalizable:

$$
a= \pm i \sqrt{b c}, \quad|a|^{2}+|b|^{2}+|c|^{2} \neq 0,
$$

where $i:=\sqrt{-1}$. In the latter case, according to (6), $H$ has the form $H=\left|\psi_{1}\right\rangle\left\langle\phi_{2}\right|$ where $\left\{\left|\psi_{a}\right\rangle,\left|\phi_{a}\right\rangle\right\}$ with $a=\{1,2\}$ is a complete biorthonormal system in $\mathrm{C}^{2}$. In particular, we have the following.

Proposition 1: Every traceless nondiagonalizable $2 \times 2$ matrix $H$ is pseudo-Hermitian.

Proof: Because $H$ is both traceless and nondiagonalizable, zero is the only eigenvalue of $H$. Hence according to Theorem 1, it must be pseudo-Hermitian.

Theorem 3: A traceless $2 \times 2$ matrix $H$ is pseudo-Hermitian if and only if it has a real determinant, i.e.,

$$
\mathcal{M}^{\prime}=\left\{H \in M_{0} \mid \operatorname{det} H \in \mathbb{R}\right\} .
$$

Proof: If $H$ is not diagonalizable, then according to Proposition 1 it is pseudo-Hermitian, and the statement of Theorem 3 is trivially satisfied. If $H$ is diagonalizable, it is either identically zero, in which case it is pseudo-Hermitian and has a real (zero) determinant, or it has two nondegenerate eigenvalues. In the latter case, in view of a proposition proven in Ref. 4, the reality of the determinant of $H$ implies its pseudo-Hermiticity. The converse is also true. For if $H$ is pseudoHermitian, then its eigenvalues are either both real or they are complex-conjugate of one another. Because $H$ has a vanishing trace, in the latter case the eigenvalues must be imaginary. This in turn implies that in both cases the determinant of $H$ is real.

In light of Theorem 3, the possibility (20) that at a level-crossing a traceless pseudo-Hermitian $2 \times 2$ matrix Hamiltonian remains diagonalizable corresponds to a single point in the uncountably infinite set of traceless nondiagonalizable pseudo-Hermitian $2 \times 2$ matrices $\mathcal{M}^{\prime}-\mathcal{M}$. To make this observation more transparent, consider the pseudo-Hermitian matrix Hamiltonians (19) corresponding to the choice $c=0$. Then $\operatorname{det} H=-a^{2}, a \in L^{ \pm}$, and $H \in \mathcal{M}^{ \pm}$. Now suppose that $a$ and $b$ are analytic functions of a real perturbation parameter $\lambda$ and that a level-crossing occurs at $\lambda$ $=0$. Then at the vicinity of the level-crossing, i.e., for $|\lambda|<\epsilon$ for some sufficiently small $\epsilon$ $\in \mathbb{R}^{+}$,

$$
a(\lambda) \approx\left\{\begin{array}{ccc}
a_{\mathrm{r}} \lambda, & \text { for } \quad-\epsilon<\lambda \leqslant 0 \\
i a_{\mathrm{i}} \lambda, & \text { for } \quad 0 \leqslant \lambda<\epsilon
\end{array}, \quad b(\lambda) \approx b_{0}+b_{1} \lambda,\right.
$$

where $a_{\mathrm{r}}$ and $a_{\mathrm{i}}$ are nonzero real constants, and $b_{0}$ and $b_{1}$ are complex constants. At $\lambda=0, H$ vanishes identically provided that $b_{0}=b(0)=0$. This is the only way in which $H$ can maintain its diagonalizability. Clearly, for $b_{0} \neq 0, H$ becomes nondiagonalizable at $\lambda=0$. In both cases $a(0)$ $=0 \in \mathbb{R}$ is the only eigenvalue (alternatively $\operatorname{det} H=0$ ). Hence, according to Corollary 1 (respectively, Theorem 3), $H$ remains pseudo-Hermitian at $\lambda=0$. This example clearly shows that the loss of diagonalizability at the crossing of the complex-conjugate eigenvalues is a generic behavior. 


\section{APPLICATION}

Consider the Wheeler-DeWitt equation for the closed FRW minisuperspace model with a real massive scalar field,

$$
\left[-\frac{\partial^{2}}{\partial \alpha^{2}}+\frac{\partial^{2}}{\partial \varphi^{2}}+e^{4 \alpha}-m^{2} e^{6 \alpha} \varphi^{2}\right] \psi(\alpha, \varphi)=0
$$

where $\alpha:=\ln a$, " $a$ " is the scale factor, $\varphi$ is a real scalar field of mass $m$, and we have chosen a particularly simple factor ordering and the natural units. ${ }^{13,14}$ The Wheeler-DeWitt equation (22) can be written in the Schrödinger form $i \dot{\Psi}=H \Psi$ where $\Psi$ is the two-component wave function, ${ }^{7}$

$$
\Psi:=\frac{1}{\sqrt{2}}\left(\begin{array}{c}
\psi+i \dot{\psi} \\
\psi-i \dot{\psi}
\end{array}\right)
$$

$H$ is the effective Hamiltonian,

$$
H:=\frac{1}{2}\left(\begin{array}{cc}
1+D & -1+D \\
1-D & -1-D
\end{array}\right)
$$

a dot means a derivative with respect to $\alpha$, and

$$
D:=-\frac{\partial^{2}}{\partial \varphi^{2}}+m^{2} e^{6 \alpha} \varphi^{2}-e^{4 \alpha} .
$$

The eigenvalue problem for the Hamiltonian (23) may be easily solved. ${ }^{7}$ The eigenvectors $\Psi_{n \pm}$ and the corresponding eigenvalues $E_{n \pm}$ have the form

$$
\Psi_{n \pm}=\frac{1}{\sqrt{2}}\left(\begin{array}{c}
1+E_{n \pm} \\
1-E_{n \pm}
\end{array}\right) \phi_{n}, \quad E_{n \pm}= \pm \sqrt{m e^{3 \alpha}(2 n+1)-e^{4 \alpha}}= \pm a \sqrt{a[m(2 n+1)-a]}
$$

where $n=0,1,2, \ldots, \phi_{n}:=N_{n} H_{n}\left(m^{1 / 2} e^{3 \alpha / 2} \varphi\right) e^{-m e^{3 \alpha} \varphi^{2} / 2}, H_{n}$ are Hermite polynomials, and $N_{n}$ $:=\left[m e^{3 \alpha} /\left(\pi 2^{2 n} n !^{2}\right)\right]^{1 / 4}$ are normalization constants.

As seen from (25), for a $\leqslant m$ the spectrum of $H$ is real, and for a $>m$ it consists of real and complex-conjugate pairs of eigenvalues. In general $H$ is pseudo-Hermitian, because $H^{\dagger}$ $=\sigma_{3} H \sigma_{3}$. For $a \neq(2 n+1) m$, it is also diagonalizable. But at the critical values $a=(2 n+1) m$ where a real (namely the zero) eigenvalue splits into a complex-conjugate pair of eigenvalues or the converse happens, $H$ fails to be diagonalizable. The situation is precisely like the one discussed in Sec. IV. Here the perturbation parameter has the form $\lambda:=a-(2 n+1) m$. At the vicinity of a level-crossing where $\lambda \rightarrow 0$, the operator $D$ and its eigenvectors do not undergo any discontinuous changes. Therefore, one can approximate the span of the eigenvectors $\Psi_{n-}$ and $\Psi_{n+}$ for $\lambda \neq 0$ with the span of the vectors,

$$
|1\rangle=\left(\begin{array}{c}
\phi_{n} \\
0
\end{array}\right), \quad|2\rangle=\left(\begin{array}{c}
0 \\
\phi_{n}
\end{array}\right),
$$

where $\phi_{n}$ is evaluated at $\alpha=\ln a=\ln [(2 n+1) m]$, i.e., $\lambda=0$. Clearly, we can study the level-crossing by confining our attention to this subspace. The above approximation becomes exact in the limit $\lambda \rightarrow 0$. In the subspace spanned by $|1\rangle$ and $|2\rangle$ the operator $D$ is identically zero. Therefore, we can approximate $D$ by a constant that tends to zero as $\lambda \rightarrow 0$. Therefore, the Hamiltonian (23) takes the form of the matrix Hamiltonian (19) with $a=(1+D) / 2, b=(-1$ $+D) / 2$, and $c=(1-D) / 2$. In the limit $\lambda \rightarrow 0, D$ approaches zero, and the conditions (21) hold. Hence, as expected, $H$ becomes nondiagonalizable at the level-crossing. 
The above argument implies that in general $H$ is diagonalizable for all values of the scale factor except the critical values $a=(2 n+1) m$. At these values $H$ becomes nondiagonalizable as one of its eigenvalues, namely the zero eigenvalue, becomes defective. The algebraic multiplicity of this eigenvalue is two. In fact, the effective Hamiltonian (23) belongs to the class of blockdiagonalizable Hamiltonians discussed in Secs. II and III. Its canonical Jordan form consists of a $2 \times 2$ Jordan block corresponding to the zero eigenvalue and an infinite number of trivial (1 $\times 1$ ) blocks corresponding to nonzero eigenvalues. The fact that this Hamiltonian is pseudoHermitian for all values of the scale factor, its spectrum consists of real and complex- conjugate eigenvalues, and its complex eigenvalues are not defective is consistent with the general results of Sec. III.

\section{SUMMARY AND CONCLUSION}

In this article we generalized our earlier results on diagonalizable pseudo-Hermitian Hamiltonians to a broad class of nondiagonalizable Hamiltonians. We showed that if a pseudo-Hermitian Hamiltonian may be mapped to a block-diagonal operator with finite-dimensional blocks via a similarity transformation, then the characterization theorems of Ref. 1 apply provided that the number and size of the Jordan blocks for the complex-conjugate pairs of eigenvalues are identical.

We also discussed the implications of our findings for the phenomenon of the loss of diagonalizability at the crossing of the complex-conjugate pairs of eigenvalues of diagonalizable pseudo-Hermitian Hamiltonians. For the latter, pseudo-Hermiticity is known to be equivalent to the presence of an antilinear symmetry. ${ }^{3}$ This in particular means that our results are relevant in the description of the PT-symmetric systems that are diagonalizable except in the case of level-crossings of the complex-conjugate eigenvalues due to perturbations of the Hamiltonian. If at the critical values of the perturbation parameter each level-crossing involves a finite number of levels, then our results apply generally. This seems to be the case for various PT-symmetric models studied in the literature. Specifically, at the critical values of the parameters of the PTsymmetric systems that undergo a spontaneous PT-symmetry breaking, a pair of real eigenvalues merge and a loss of diagonalizability similar to the one discussed in Sec. V occurs.

As a final note, we wish to emphasize that the results of this paper rely on the basic assumption that the quantum system has a genuine separable Hilbert space in which the Hamiltonian acts. For many PT-symmetric Hamiltonians the (inner product) structure of the function space in which one solves for the eigenfunctions is not clear. In this context, the assumption of considering non-Hermitian Hamiltonians acting in a separable Hilbert space may seem too restrictive. Nevertheless, we believe that this assumption provides a framework for exploring some of the intriguing properties of a class of non-Hermitian Hamiltonians. This class includes many PT-symmetric Hamiltonians as well as all the matrix Hamiltonians and the non-Hermitian Hamiltonians appearing in the two-component formulation of the Klein-Gordon and Wheeler-DeWitt equations.

\section{ACKNOWLEDGMENTS}

I wish to thank the anonymous referee(s) of Refs. 3 and 4, who emphasized that the diagonalizability assumption made in these papers might be a serious limitation, and M. Znojil who convinced me that a loss of diagonalizability occured in certain PT-symmetric models. Finally I would like to acknowledge the support of the Turkish Academy of Sciences through the Young Researcher Award Program (GEBIP).

\footnotetext{
${ }^{1}$ A. Mostafazadeh, J. Math. Phys. 43, 205 (2002).

${ }^{2}$ A. Mostafazadeh, J. Math. Phys. 43, 2814 (2002).

${ }^{3}$ A. Mostafazadeh, J. Math. Phys. 43, 3944 (2002).

${ }^{4}$ A. Mostafazadeh, Nucl. Phys. B 640, 419 (2002).

${ }^{5}$ A. Mostafazadeh, "A genuine probability interpretation for Klein-Gordon fields and its implications for quantum cosmology," arXiv: gr-qc/0205049.

${ }^{6}$ C. M. Bender and S. Boettcher, Phys. Rev. Lett. 80, 5243 (1998); F. M. Fernández, R. Guardiola, J. Ros, and M. Znojil, J. Phys. A 31, 10105 (1998); F. Cannata, G. Junker, and J. Trost, Phys. Lett. A 246, 219 (1998); C. M. Bender, S.
} 
Boettcher, and P. N. Meisenger, J. Math. Phys. 40, 2201 (1999); P. Dorey, "The ODE/IM correspondence and PTsymmetric quantum mechanics," arXiv: hep-th/0201108 and references therein.

${ }^{7}$ A. Mostafazadeh, J. Math. Phys. 39, 4499 (1998).

${ }^{8}$ Z. Ahmed, Phys. Lett. A 290, 19 (2001); 294, 287 (2002); S. M. Klishevich and M. Plyushchay, Nucl. Phys. B 628, 217 (2002); L. Solombrino, "Weak pseudo-Hermiticity and antilinear commutant," arXiv: quant-ph/0203101; B. Bagchi and C. Quesne, "Non-Hermitian Hamiltonians with real and complex eigenvalues in a Lie-algebraic framework," arXiv: quant-ph/0205002.

${ }^{9}$ A. Mostafazadeh, "On the pseudo-Hermiticity of general PT-symmetric standard Hamiltonians in one dimension," arXiv: math-ph/0204013.

${ }^{10}$ V. V. Prasolov, Problems and Theorems in Linear Algebra (American Mathematical Society, Providence, RI, 1994).

${ }^{11}$ T. Kato, Perturbation Theory for Linear Operators (Springer-Verlag, Berlin, 1995).

${ }^{12} \mathrm{M}$. Znojil (private communications).

${ }^{13}$ D. N. Page, in Gravitation: A Banff Summer Institute, edited by R. Mann and P. Wesson (World Scientific, Singapore, 1991).

${ }^{14}$ D. L. Wiltshire, in Cosmology: The Physics of the Universe, edited by B. Robson, N. Visvanathan, and W. S. Woolcock (World Scientific, Singapore, 1996). 